\title{
Recurrence Coefficients of Orthogonal Polynomials with Respect to Some Self-Similar Singular Distributions
}

\author{
H.-J. Fischer
}

\begin{abstract}
The limiting behaviour of arithmetic and geometric means of the coefficients of three term recurrence relations satisfied by orthogonal polynomials is investigated. The measure of orthogonality is not assumed to be absolutely continuous, but it must guarantee regular limit distribution of the zeros of the orthogonal polynomials. Some examples of self-similar distributions satisfying this condition are given.
\end{abstract}

Keywords: Orthogonal polynomials, recurrence relations, zero distribution

AMS subject classification: $43 \mathrm{C} 05$

\section{Introduction}

In the last years it has been realized that singular measures are not only pathological examples but have important applications in such areas as theoretical physics or others (see [1] and the references cited there). Consequently, there has been some interest in the study of orthogonal polynomials with respect to these measures, especially of their three term recurrence relation.

First, we introduce some notations. Let $\mu$ be a finite Borel measure on the real line, with support (or spectrum)

$$
S=S(\mu)=\{x \in \mathbf{R}: \mu([x-\delta, x+\delta])>0 \text { for all } \delta>0\}
$$

We assume $S$ to be an infinite compact set. Then there exist unique orthonormal polynomials

$$
p_{n}(x)=\gamma_{n} x^{n}+\ldots \quad\left(\gamma_{n}=\gamma_{n}(\mu)>0\right)
$$

with orthogonality relation

$$
\int p_{m}(x) p_{n}(x) d \mu(x)=\delta_{m n} \quad(m, n \geq 0)
$$

The corresponding monic (i.e. with leading coefficient 1) polynomial of degree $n$ we denote by $P_{n}$. Obviously, $P_{n}=\frac{1}{\gamma_{n}} p_{n}$. The recurrence relation takes the form

$$
P_{n}(x)=\left(x-c_{n}\right) P_{n-1}(x)-\lambda_{n} P_{n-2}(x) \quad(n \geq 1)
$$

H.-J. Fischer: Techn. Univ. Chemnitz-Zwickau, Fak. Math., PSF 964, D - 09009 Chemnitz 
where we assume $P_{-1}=0$ and $P_{0}=1$. In this relation the coefficient $\lambda_{1}$ would be arbitrary, but it is often convenient to assume $\lambda_{1}=\mu(S)$, since with this convention the normalization constant simply writes

$$
\frac{1}{\gamma_{n}}=\left(\lambda_{1} \ldots \lambda_{n+1}\right)^{1 / 2}
$$

(for these and other simple facts about general orthogonal polynomials we refer to [2: Chapter I]). The asymptotic behaviour of the coefficients $c_{n}$ and $\lambda_{n}$ is very interesting. A well known result of Rakhmanov states the following:

$$
\begin{aligned}
& \text { If } S \subset[0,1] \text { and } \frac{d \mu(x)}{d x}>0 \text { a.e. on }[0,1] \text {, then } \\
& \qquad \lim _{n \rightarrow \infty} c_{n}=\frac{1}{2} \quad \text { and } \quad \lim _{n \rightarrow \infty} \lambda_{n}=\frac{1}{16}
\end{aligned}
$$

(the assumptions, of course, imply $S=[0,1])$. For the singular measures considered in [1], the coefficients do not converge. However, in [4] it is suggested to investigate the behaviour of the arithmetic means

$$
\frac{1}{n-1} \sum_{k=2}^{n} \lambda_{k}
$$

though in view of (1) the geometric mean would be more natural. In the case of an almost periodic sequence the arithmetic means, of course, converge.

In the present note, we show that under appropriate assumptions the limits

$$
\lim _{n \rightarrow \infty} \frac{1}{n} \sum_{k=1}^{n} c_{k}, \quad \lim _{n \rightarrow \infty} \frac{1}{n}\left(\sum_{k=1}^{n} c_{k}^{2}+2 \sum_{k=2}^{n} \lambda_{k}\right), \quad \lim _{n \rightarrow \infty}\left(\lambda_{2} \ldots \lambda_{n}\right)^{1 / n}
$$

exist. In the case $S=[0,1]$, we can prove a weaker version of (2), which we could call convergence in density.

Our results. in some sense complement the investigations of Mercer [5], wherc the reverse problem is investigated: If we are given the mean behaviour of the sequences $\left(c_{n}\right)$ and $\left(\lambda_{n}\right)$ - what can we say about the limiting distribution of the zeros of the polynomials $p_{n}$ ?

\section{Connection with zero distribution}

There is a simple relation between two of the above expressions in (4) and the zeros of the polynomial $p_{n}$.

Lemma 1. Let $x_{n 1}, \ldots, x_{n n}$ be the zeros of $p_{n}$. Then

$$
\sum_{k=1}^{n} x_{n k}=\sum_{k=1}^{n} c_{k}
$$


and

$$
\sum_{k=1}^{n} x_{n k}^{2}=\sum_{k=1}^{n} c_{k}^{2}+2 \sum_{k=2}^{n} \lambda_{k}
$$

Proof. Since the zeros of the polynomials $p_{n}$ and $P_{n}$ coincide, we have

$$
P_{n}(x)=\left(x-x_{n 1}\right) \ldots\left(x-x_{n n}\right)
$$

Thus the coefficient of $x^{n-1}$ in $P_{n}(x)$ is $-\left(x_{n 1}+\ldots+x_{n n}\right)$. On the other hand, this coefficient is equal to $-\left(c_{1}+\ldots+c_{n}\right)$ (see [2: p. 19]), and this proves (5).

The coefficient of $x^{n-2}$ is $\sum_{1 \leq i<j \leq n} x_{n i} x_{n}$; and must be equal to

$$
\sum_{1 \leq i<j \leq n} c_{i} c_{j}-\sum_{k=2}^{n} \lambda_{k}
$$

(see [2: p. 24]). From this we have the elementary identity

$$
\begin{aligned}
\sum_{k=1}^{n} x_{n k}^{2} & =\left(\sum_{k=1}^{n} x_{n k}\right)^{2}-2 \sum_{1 \leq i<j \leq n} x_{n i} x_{n j} \\
& =\left(\sum_{k=1}^{n} c_{k}\right)^{2}-2\left(\sum_{1 \leq i<j \leq n} c_{i} c_{j}-\sum_{k=2}^{n} \lambda_{k}\right) \\
& =\sum_{k=1}^{n} c_{k}^{2}+2 \sum_{k=2}^{n} \lambda_{k}
\end{aligned}
$$

and (6) is verified.

Remark. It would be difficult (though possible) to write down the corresponding identities for $\sum_{k=1}^{n} x_{n k}^{m}$ with $m \geq 3$. In fact, we do not need the exact equalities to study limits of mean values, since the following fact of $P$. Nevai holds (this is [6: Lemma 5.1]).

If the support $S(\mu)$ is compact and the function $f$ is continuous, then

$$
\lim _{n \rightarrow \infty} \frac{1}{n} \sum_{k=1}^{n}\left\{f\left(x_{n k}\right)-\int f(x) p_{k-1}^{2}(x) d \mu(x)\right\}=0 .
$$

This could replace (with $f(x)=x$ or $f(x)=x^{2}$ ) the equations (5) and (6), but the above derivation is simpler.

The existence of the limits

$$
\lim _{n \rightarrow \infty} \frac{1}{n} \sum_{k=1}^{n} x_{n k} \quad \text { and } \quad \lim _{n \rightarrow \infty} \frac{1}{n} \sum_{k=1}^{n} x_{n k}^{2}
$$


or, more general,

$$
\lim _{n \rightarrow \infty} \frac{1}{n} \sum_{k=1}^{n} f\left(x_{n k}\right)
$$

for any continuous function $f$ on $S(\mu)$ depends on wether the zeros of the polynomial $p_{n}$ have a regular limiting behaviour in the sense of Ullman [9], i.e. wether $\mu$ is a regular measure in the sense of [9] or [8]. Some sufficient conditions for regularity will be cited in the next section. We only mention that absolute continuity of the measure $\mu$ (and moreover positivity a.e. of the density) is not necessary. In fact already in [9] an example of an atomic regular measure is given.

\section{Regular measures}

For the following we need some facts from potential theory (see [8] or [7]). For a probability measure $\nu$ with compact support $S(\nu)$ in the complex plane $\mathbf{C}$ the logarithmic energy of $\nu$ is defined as

$$
I(\nu)=\iint \log \frac{1}{|z-t|} d \nu(z) d \nu(t) .
$$

If $K$ is a compact subset of $\mathbf{C}$, then with

$$
V_{K}=\inf _{S(\nu) \subset K} I(\nu)
$$

the capacity of $K$ is defined as

$$
\operatorname{cap} K=\exp \left(-V_{K}\right)
$$

If cap $K>0$, then there exists a unique probability measure $\omega_{K}$, called the equilibrium distribution of $K$, at which the infimum in (7) is attained. The notion of capacity can be extended to any Borel set $B$ : $\operatorname{cap} B$ denotes the supremum of the capacities of compact subsets of $B$. We say that a property holds q.e. (quasi everywhere) on a set $S \subset \mathbf{C}$ if it holds on $S$ with possible exceptions on a subset of capacity zero.

Now we are able to define the notion of regularity of the measure: A measure $\mu$ with support $S=S(\mu)$ is called regular if

$$
\lim _{n \rightarrow \infty} \gamma_{n}(\mu)^{1 / n}=\frac{1}{\operatorname{cap} S(\mu)}
$$

As it is shown in [8], there are several equivalent formulations of regularity. We list only a few of them:

1. $\limsup \left|p_{n}(x)\right|^{1 / n}=1$ for quasi-every $x \in S(\mu)$ (see [8: Theorem 3.1/(iii)]; this is essentially the notion of regularity in [9]).

2. $\underset{n \rightarrow \infty}{\limsup }\left|p_{n}(x)\right|^{1 / n} \leq 1$ for quasi-every $x \in S(\mu)$ (see [8: Theorem 3.2/(iv)]). 
3. $\lim _{n \rightarrow \infty}\left(\sup _{x \in S(\mu)}\left|p_{n}(x)\right|\right)^{1 / n} \leq 1$ (if $S(\mu)$ is regular with respect to Dirichlet problems in $\mathrm{C} \backslash S(\mu)$, see [8: Theorem 3.3/(iv)]).

Of course, the definition (8) is just what we would like to prove for a measure, i.e. we need some easy to verify criteria for the regularity of a measure. Some of them can be found in [8]. For our purposes their "criterion $\Lambda$ " suffices: Let $\Delta_{r}(x)=\{z \in \mathbf{R}$ : $|z-x| \leq r\}$. Then

$$
\operatorname{cap}\left\{x \in \mathbf{R}: \limsup _{r \rightarrow 0+} \frac{\log 1 / \mu\left(\Delta_{r}(x)\right)}{\log 1 / r}<\infty\right\}=\operatorname{cap} S(\mu)
$$

implies the regularity of the measure $\mu$ (see [8: Theorem 4.8]). Since regularity also implies regular limiting zero distribution, we can state our first result.

Theorem 1. Let $\mu$ be a regular measure, $S$ its support and $\omega_{S}$ its equilibrium distribution. Then

$$
\begin{aligned}
\lim _{n \rightarrow \infty} \frac{1}{n} \sum_{k=1}^{n} c_{k} & =\int x d \omega_{S}(x) \\
\lim _{n \rightarrow \infty} \frac{1}{n}\left(\sum_{k=1}^{n} c_{k}^{2}+2 \sum_{k=2}^{n} \lambda_{k}\right) & =\int x^{2} d \omega_{S}(x) \\
\lim _{n \rightarrow \infty}\left(\lambda_{2} \ldots \lambda_{n}\right)^{1 / n} & =(\operatorname{cap} S)^{2}
\end{aligned}
$$

Proof. For any $n>0$ let $\nu_{n}$ denote the measure that places mass 1 to every zero of $p_{n}$. The regularity of $\mu$ implies (see [8: Theorem 5.8/(a)])

$$
\frac{1}{n} \nu_{n} \stackrel{*}{\longrightarrow} \omega_{S} \quad \text { for } n \rightarrow \infty
$$

and this means

$$
\lim _{n \rightarrow \infty} \frac{1}{\dot{n}} \int f(x) d \nu_{n}(x)=\lim _{n \rightarrow \infty} \frac{1}{n} \sum_{k=1}^{n} f\left(x_{n k}\right)=\int f(x) d \omega_{S}(x)
$$

for any continuous function $f$ on $S$. Now for $f(x)=x$ with (5) we obtain (10), for $f(x)=x^{2}$ with (6) we obtain (11). The third limit (12) is an immediate consequence of (1) and (8).

Let us illustrate this theorem by two examples.

3.1 Bessis example. In his article [1], D. Bessis investigates orthogonal polynomials with respect to the equilibrium distribution of the Julia set of a polynomial $T=T(z)$. In general, this is a set in the complex plane. In the special case $T(z)=z^{2}-\lambda$ with $\lambda$ real and $\lambda>2$, he shows that the Julia set is a Cantor type set on the real line $K \subset[-\xi, \xi]$, where

$$
\xi=\frac{1+\sqrt{1+4 \lambda}}{2}
$$


(one easily shows $2<\xi<\lambda$ ). Here $\mu=\omega_{K}$ is symmetric and invariant under the transformation $T(x)=x^{2}-\lambda$, and we obtain

$$
\int x d \omega_{K}(x)=0 \quad \text { and } \quad \int\left(x^{2}-\lambda\right) d \omega_{K}(x)=\int x d \omega_{K}(x)=0 .
$$

Consequently,

$$
\int x^{2} d \omega_{K}(x)=\int \lambda d \omega_{K}(x)=\lambda .
$$

The regularity of $\mu$ follows from the explicit bound

$$
\left|p_{n}(x)\right|<\frac{1}{2}\left(\frac{4 \lambda^{1 / 2}}{\sqrt{1+4 \lambda}-1}\right)^{k} \text { for } 2^{k-1} \leq n^{\prime} \leq 2^{k}
$$

when $x \in K$ (this is [1: eq. IV:141]), since this implies

$$
\limsup _{n \rightarrow \infty}\left|p_{n}(x)\right|^{1 / n} \leq 1 \quad \text { for } x \in K
$$

one of the equivalent formulations of regularity. Thus our Theorem 1 is applicable, but we need the capacity of $K$. Implicitely, it is calculated in [1, eq. II.35]: if we compare this with the general formula for the Green function (e.g. [8: p. 397]), we see that $\log \operatorname{cap} K=0$, i.e. $\operatorname{cap} K=1$. For the symmetric measure $\mu$, of course, all $c_{n}$ are zero, so. (10) is trivial, and (11) becomes

$$
\lim _{n \rightarrow \infty} \frac{1}{n} \sum_{k=2}^{n} \lambda_{k}=\frac{\lambda}{2}
$$

Equation (12) takes the form

$$
\lim _{n \rightarrow \infty}\left(\lambda_{2} \ldots \lambda_{n}\right)^{1 / n}=1
$$

We can now compare our results with the recurrences derived by Bessis: his system of equations [1: IV.105] in our notations reads

$$
\begin{array}{r}
\lambda_{2 k-1} \lambda_{2 k-2}=\lambda_{k} \\
\lambda_{2 k-1}+\lambda_{2 k}=\lambda \\
\vdots \quad \lambda_{2}=\lambda
\end{array}
$$

for $k \geq 2$. Equation (14) follows from (15) easily (if the existence of the limit is known), (16) immediately gives (13). Observe that for a measure satisfying. the conditions of Rakhmanov:on the interval $[-\xi, \xi]$ we would have (after an obvious rescaling) $\lim _{n \rightarrow \infty} \lambda_{n}=\xi^{2} / 4$, which is strictly between 1 and $\lambda / 2\left(\right.$ since $\xi^{2}=\xi+\lambda$ and $\left.2<\xi<\lambda\right)$. 
3.2 Cantor measure. In the article :[4] moments of the: Cantor measure (tógether with interesting asymptotics) are investigated and the first recurrence coefficients $\lambda_{n}$ are generated. We will not give a detailed construction of the Cantor set and the Cantor measure here, but only recall a few facts sufficient to prove the regularity of the measure.

The Cantor set $C$ is defined by

$$
C=\bigcap_{n=0}^{\infty} \bigcup_{k=1}^{2^{n}} I_{n k}
$$

where for any $n \geq 0$ the sets $I_{n k} \subset[0,1]$ are disjoint intervals of length $3^{-n}$. The Cantor measure $\mu$ is defined by

$$
\mu\left(I_{n k}\right)=\frac{1}{2^{n}}
$$

Let $3^{-n} \leq r \leq 3^{-n+1}$ and $x \in C$. This means that there is a $k=k(x)$ with $x \in I_{n k}$. Since the length of $I_{n k}$ is $3^{-n} \leq r$, we have

$$
\Delta_{r}(x) \supset I_{n k} \quad \text { and } \quad \mu\left(\Delta_{r}(x)\right) \geq \mu\left(I_{n k}\right)=\frac{1}{2^{n}} \geq\left(\frac{r}{3}\right)^{\alpha}
$$

where $\alpha=\log 2 / \log 3$ : From this

$$
\limsup _{r \rightarrow 0+} \frac{\log 1 / \mu\left(\Delta_{r}(x)\right)}{\log 1 / r} \leq \alpha<\infty
$$

follows for all $x \in C$ (not only q.e.), and criterion $\Lambda$ (9) is satisfied. Thus we have proved the following

Lemma 2.: The Cantor measure $\mu$ is regular.

The measure $\mu$ is symmetric about $\frac{1}{2}$, hence $c_{n} \equiv \frac{1}{2}$ and (10) is again trivial. The statements (11) and (12) of our Theorem 1 now give the following

Corollary 1. The recurrence coefficients $\lambda_{n}$ "for the Cantor measure satisfy the relations

$$
\lim _{n \rightarrow \infty} \frac{1}{n} \cdot \sum_{k=2}^{n} \lambda_{k}=\frac{1}{2}\left(\int x^{2} d \omega_{C}(x)-\frac{1}{4}\right) \ldots
$$

and

$$
\lim _{n \rightarrow \infty}\left(\lambda_{2} \ldots \lambda_{n}\right)^{1 / n}=(\operatorname{cap} C)^{2} \because
$$

Unfortunately, this is far less explicit than (13) and (14), since cap $C$ and $\omega_{C}$ are unknown (to the author). 


\section{Dilation, translation and symmetrization of a measure}

To prove stronger results in the case $S=[a, b]$, we need a device to reduce this situation to a simple standard case (e.g. $S=[0,1]$ ). If $\mu$ is a measure. with support $S(\mu)=[a, b]$, then we can define a new measure $\mu_{1}$ via

$$
\int f(a+(b-a) x) d \mu_{1}(x)=\int f(x) d \mu(x)
$$

for any measurable bounded function $f$ on $[a, b]$. Obviously, $S\left(\mu_{1}\right)=[0,1]$, and if $P_{n}(n \geq 0)$ are the monic orthogonal polynomials with respect to $\mu$, then

$$
P_{n}(a+(b-a) x) \quad(n \geq 0)
$$

must be orthogonal with respect to $\mu_{1}$. Since $P_{n}(a+(b-a) x)$ has leading coefficient $(b-a)^{n}$, the monic orthogonal polynomials with respect to $\mu_{1}$ must be

$$
Q_{n}(x)=(b-a)^{-n} P_{n}(a+(b-a) x) .
$$

Solving for $P_{n}$ and substituting this into the recurrence relation (with $x$ replaced by $a+(b-a) x)$ immediately gives

$$
Q_{n}(x)=\left(x-\frac{c_{n}(\mu)-a}{b-a}\right) Q_{n-1}(x)-\frac{\lambda_{n}(\mu)}{(b-a)^{2}} Q_{n-2}(x)
$$

for $n \geq 1$ (this is essentially [2: Chapter I, Exercise 4.4/(a)]). Consequently, the recurrence coefficients corresponding to the measures $\mu$ and $\mu_{1}$ are connected via the equations

$$
c_{n}\left(\mu_{1}\right)=\frac{c_{n}(\mu)-a}{b-a} \cdot \quad \text { and } \quad \lambda_{n}\left(\mu_{1}\right)=\frac{\lambda_{n}(\mu)}{(b-a)^{2}}
$$

where the second equation, of course, is valid only for $n \geq 2$ (since $Q_{-1}=0$ ).

Furthermore, we should be able to transform information about the coefficients $\lambda_{n}$ alone into statements concerning both $c_{n}$ and $\lambda_{n}$. In the case of symmetric (about zero) measures we have, of course, $c_{n} \doteq 0$. But the general case can be reduced to this! Let $\mu$ be any measure with $S(\mu) \subset[0, \infty)$ (we always can achieve this by an appropriate shift). We define a measure $\nu$ by

$$
\int x^{2 n} d \nu(x)=\int x^{n} d \mu(x) . \text { and } \int x^{2 n+1} d \nu(x)=0
$$

for $n \geq 0$ (we assumed $S(\mu)$ to be a compact set, so $\nu$ is uniquely determined). Then the monic orthogonal polynomials $S_{n}$ with respect to $\nu$ are

$$
S_{2 n}(x)=P_{n}\left(x^{2}\right) \quad \text { and } . \quad S_{2 n+1}(x)=x P_{n}^{*}\left(0 ; x^{2}\right)
$$

for $n \geq 0$, where

$$
P_{n}^{*}(0 ; x)=\frac{1}{x}\left(P_{n+1}(x)-\frac{P_{n+1}(0)}{P_{n}(0)} P_{n}(x)\right)
$$


(this is [2: Chapter I, Theorem 8.1]). There is a simple relation between the recurrence coefficients, too: The coefficients $c_{n}(\nu)$ are identically zero by symmetry, and the $\lambda_{n}(\nu)$ satisfy

$$
\begin{aligned}
& c_{1}(\mu)=\lambda_{2}(\nu) \\
& c_{n}(\mu)=\lambda_{2 n-1}(\nu)+\lambda_{2 n}(\nu) \quad(n \geq 2) \\
& \lambda_{n}(\mu)=\lambda_{2 n-2}(\nu) \lambda_{2 n-1}(\nu) \quad(n \geq 2)
\end{aligned}
$$

(see [2: Chapter I, Theorem 9.1]).

\section{The case $S=[a, b]$}

We could now specify our result to the case $S=[a, b]$, where

$$
\operatorname{cap} S=\frac{b-a}{4} \quad \text { and } . d \omega_{S}(x)=\frac{1}{\pi \sqrt{(b-x)(x-a)}} d x \text {, }
$$

but instead we shall prove a stronger theorem in a more direct way. Using dilations and translations, we can reduce our problem to the case $S=[0,1]$. The general case will be obtained from the equations (20). First we need some elementary estimates.

Lemma 3. Let $S=[0,1]$. Then for the recurrence coefficients $\lambda_{n}$ we have the inequality

$$
\lambda_{2}^{1 / 2}+\ldots+\lambda_{n}^{1 / 2} \leq \frac{n}{4} .
$$

Proof. It is well known that the zeros of $p_{n}$ are the eigenvalues of the Jacobi matrix

$$
J_{n}=\left(\begin{array}{ccccc}
c_{1} & \lambda_{2}^{1 / 2} & & & 0 \\
\lambda_{2}^{1 / 2} & c_{2} & \lambda_{3}^{1 / 2} & & \\
& \ddots & \ddots & \ddots & \\
& & \lambda_{n-1}^{1 / 2} & c_{n-1} & \lambda_{n}^{1 / 2} \\
0 & & & \lambda_{n}^{1 / 2} & c_{n}
\end{array}\right)
$$

These are all in the interval $(0,1)$ and consequently

$$
0 \leq\left\langle x, J_{n} x\right\rangle \leq\langle x, x\rangle \quad \text { for any } x \in \mathbf{R}^{n} .
$$

From the first inequality with $x=(1,-1,1, \ldots)^{T}$ we obtain

$$
\sum_{k=1}^{n} c_{k}-2 \sum_{k=2}^{n} \lambda_{k}^{1 / 2} \geq 0
$$

from the second with $x=(1,1,1, \ldots)^{T}$ we obtain

$$
\sum_{k=1}^{n} c_{k}+2 \sum_{k=2}^{n} \lambda_{k}^{1 / 2} \leq n
$$

Subtracting these two inequalities and dividing by 4 we arrive at (25). 
Lemma 4. Let $S(\mu)=[0,1]$ and $M_{n}=\sup _{x \in[0,1]}\left|p_{n}(x)\right|$. Then

$$
\frac{M_{n}}{\gamma_{n}} \geq \frac{1}{2^{2 n-1}} \quad \text { for } n \geq 1
$$

Proof. We have $M_{n} / \gamma_{n}=\sup _{x \in[0,1]}\left|P_{n}(x)\right|$ (compare the proof of [7: Proposition $3.2]$ ) and thus the proposition is a consequence of the fact that of all monic polynomials the monic shifted Chebyshev polynomial has minimal supremum norm on $[0,1]$.

Lemma 5. If $S(\mu)=[0,1]$ and $M_{n}=\sup _{x \in[0,1]}\left|p_{n}(x)\right|$, then the in equality

$$
\sum_{k=2}^{n}\left(4 \lambda_{k}^{1 / 2}-1-\log 4 \lambda_{k}^{1 / 2}\right) \leq 1-\log \frac{2}{\lambda_{1}^{1 / 2}}+\log M_{n-1}
$$

holds for $n \geq 2$.

Proof. The inequality follows from (1), (26) with $n-1$ instead of $n$ and (25) just by elementary algebra

The preceeding lemma says nothing about the $c_{n}$, so we have to investigate the symmetrization $\nu$ of $\mu$, first. Of course, we must have a result like

Lemma 6. If $S(\mu)=[0 ; 1]$ and $\mu$ is regular, then $\nu$ is regular, too

Proof. Evidently, $S(\nu)=[-1,1]$. The regularity of $\mu$ by definition (8) implies

$$
\lim _{n \rightarrow \infty} \gamma_{n}(\mu)^{1 / n}=\frac{1}{\operatorname{cap} S(\mu)}=4
$$

To verify the regularity of $\nu$, we have to find a relation between $\gamma_{n}(\mu)$ and $\gamma_{n}(\nu)$. But from (1) and (23) we have

$$
\frac{1}{\gamma_{2 n}(\nu)}=\left(\lambda_{1}(\nu) \ldots \lambda_{2 n+1}(\nu)\right)^{1 / 2}=\left(\lambda_{1}(\mu) \ldots \lambda_{n+1}(\mu)\right)^{1 / 2}=\frac{1}{\gamma_{n}(\mu)}
$$

and (since all $\lambda_{n}(\nu)<\dot{1}$ )

and consequently

$$
\frac{1}{\gamma_{2 n}(\nu)} \geq \frac{1}{\gamma_{2 n+1}(\nu)} \geq \frac{1}{\gamma_{2 n+2}(\nu)}
$$

$$
\lim _{n \rightarrow \infty} \gamma_{n}(\nu)^{1 / n}=\lim _{n \rightarrow \infty} \gamma_{2 n}(\nu)^{1 / 2 n}=\lim _{n \rightarrow \infty} \gamma_{n}(\mu)^{1 / 2 n}=\sqrt{4}=2:
$$

This means the regularity of $\nu$, because of $\operatorname{cap}[-1,1]=\frac{1}{2}$.

To formulate our next result, we need some definitions. Let $M$ be a subset of the natural numbers. We call the limit

$$
d(M) \stackrel{\because}{=} \limsup _{n \rightarrow \infty} \frac{\#\{k \leq n: k \in M\}}{n}
$$

the density of $M$. We say that a sequence $\left(a_{n}\right)$ converges to $a$ in density $\left(a_{n} \stackrel{d}{\longrightarrow} a\right)$ if .

$$
d\left(\left\{n:\left|a_{n}-a\right| \geq \delta\right\}\right)=0 \text { for all } \delta>0 .
$$

This notion is weaker than convergence (in the case of convergence, the set $\left\{n:\left|a_{n}-a\right| \geq\right.$ $\delta\}$ is finite for all $\delta>0$, and a finite set has density zero). But it is meaningful, as the following lemmina shows. 
Lemma 7. $a_{n} \stackrel{d}{\longrightarrow} a$ if and only if

$$
\lim _{n \rightarrow \infty} \frac{1}{n} \sum_{k=1}^{n} f\left(a_{k}\right)=f(a)
$$

for all bounded continuous functions $f$.

Proof. Suppose first $a_{n} \stackrel{d}{\longrightarrow} a$. Then we have

$$
\left|\frac{1}{n} \sum_{k=1}^{n} f\left(a_{k}\right)-f(a)\right|=\left|\frac{1}{n} \sum_{k=1}^{n}\left(f\left(a_{k}\right)-f(a)\right)\right| \leq \frac{1}{n} \sum_{k=1}^{n}\left|f\left(a_{k}\right)-f(a)\right| .
$$

Now if $f$ is bounded, $|f(x)| \leq K$ for all $x$. Let $\varepsilon$ be any positive real number. Then there exists a $\delta>0$ with $|f(x)-f(a)|<\varepsilon$ whenever $|x-a|<\delta$. If we.write

$$
N_{\delta}=\left\{\dot{n}:\left|a_{n}-a\right| \geq \delta\right\}
$$

then we have $\left|f\left(a_{k}\right)-f(a)\right|<2 K$ for $k \in N_{\delta}$ and $\left|f\left(a_{k}\right)-f(a)\right|<\varepsilon$ for $k \notin N_{\delta}$, consequently

$$
\frac{1}{n} \sum_{k=1}^{n}\left|f\left(a_{k}\right)-f(a)\right| \leq \epsilon+\frac{2 K}{n} \#\left\{k \leq n: k \in N_{\delta}\right\}
$$

From this

$$
\limsup _{n \rightarrow \infty}\left|\frac{1}{n} \sum_{k=1}^{n} f\left(a_{k}\right)-f(a)\right| \leq \underset{n \rightarrow \infty}{\limsup } \frac{1}{n} \sum_{k=1}^{n}\left|f\left(a_{k}\right)-f(a)\right| \leq
$$

follows, i.e.

$$
\lim _{n \rightarrow \infty} \frac{1}{n} \sum_{k=1}^{n} f\left(a_{k}\right)=f(a)
$$

since $\varepsilon>0$ was arbitrary.

To prove the "if"-part, suppose (27) holds. For $\delta>0$ let $f(x)=\min \left\{\frac{1}{\delta}|x-a|, 1\right\}$. Obviously, $f$ is continuous and bounded, and with the notation(28) we have.

$$
\frac{1}{n} \sum_{k=1}^{n} f\left(a_{k}\right) \geq \frac{1}{n} \#\left\{k \leq n: k \in N_{6}\right\}
$$

The equation (27) for this special function $f$ now immediately gives $d\left(N_{\delta}\right)=0$.

The main result of this section is the following

Theorem 2. Let $\mu$ be regular and $S(\mu)=[a, b]$. Then

$$
\lambda_{n} \stackrel{d}{\longrightarrow}\left(\frac{b-a}{4}\right)^{2} \text { and } \quad c_{n} \stackrel{d}{\longrightarrow} \frac{a+b}{2} \text {. }
$$


Proof. First we assume $S(\mu)=[0,1]$. We prove the proposition concerning $\lambda_{n}$. Let again $M_{n}=\sup _{x \in[0,1]}\left|p_{n}(x)\right|$. The equivalent formulation 3 of regularity (the condition for $S(\mu)$ is satisfied) gives

$$
\lim _{n \rightarrow \infty} M_{n}^{1 / n}=1, \quad \text { i.e. } \quad \lim _{n \rightarrow \infty} \frac{1}{n} \log M_{n}=0
$$

Now we can apply our Lemma 5 . Observe the elementary inequality $\log x \leq x-1$ for $x>0$. It shows that the sum in this lemma is positive! Moreover, substituting $x^{1 / 2}$ instead of $x$, we obtain

$$
x-1-\log x \geq x-1-2\left(x^{1 / 2}-1\right)=\left(x^{1 / 2}-1\right)^{2} .
$$

Thus

$$
\begin{aligned}
\frac{1}{n} \sum_{k=2}^{n}\left(4 \lambda_{k}^{1 / 2}-1-\log 4 \lambda_{k}^{1 / 2}\right) & \geq \frac{1}{n} \sum_{k=2}^{n}\left(2 \lambda_{k}^{1 / 4}-1\right)^{2} \\
& \geq \frac{\delta^{2}}{n} \#\left\{k \leq n:\left|2 \lambda_{k}^{1 / 4}-1\right| \geq \delta\right\}
\end{aligned}
$$

for any $\delta>0$. Since the left-hand side converges to zero as $n \rightarrow \infty$ by Lemma 5 , we have $2 \lambda_{n}^{1 / 4} \stackrel{d}{\longrightarrow} 1$ and this immediately gives $\lambda_{n} \stackrel{d}{\longrightarrow} \frac{1}{16}$. Now we are going to prove the second proposition concerning $c_{n}$. To do this we consider the symmetrization $\nu$ of $\mu$. By Lemma 6 it is regular and we have $S(\nu)=[-1,1]$. What we have shown above implies $\lambda_{n}(\nu) \stackrel{d}{\longrightarrow} \frac{1}{16}[1-(-1)]^{2}=\frac{1}{4}$ in virtue of the scaling properties of recurrence coefficients (see equations (20)). From this by (22) and (23)

$$
c_{n}(\mu)=\lambda_{2 n-1}(\nu)+\lambda_{2 n}(\nu) \stackrel{d}{\longrightarrow} \frac{1}{4}+\frac{1}{4}=\frac{1}{2}
$$

follows and we have

$$
\lambda_{n}(\mu)=\lambda_{2 n-2}(\nu) \lambda_{2 n-1}(\nu) \stackrel{d}{\longrightarrow} \frac{1}{4} \cdot \frac{1}{4}=\frac{1}{16} .
$$

The general case $S=[a ; b]$ follows now easily: The corresponding shifted and scaled to $[0,1]$ measure $\mu_{1}$ defined in the preceeding section is regular, and we have $c_{n}\left(\mu_{1}\right) \stackrel{d}{\longrightarrow} \frac{1}{2}$ and $\lambda_{n}\left(\mu_{1}\right) \stackrel{d}{\longrightarrow} \frac{1}{16}$. But from the equations (20) we obtain

$$
c_{n}(\mu)=a+(b-a) c_{n}\left(\mu_{1}\right) \stackrel{d}{\longrightarrow} a+\frac{b-a}{2}=\frac{a+b}{2}
$$

and

$$
\lambda_{n}(\mu)=(b-a)^{2} \lambda_{n}\left(\mu_{1}\right) \stackrel{d}{\longrightarrow} \frac{(b-a)^{2}}{16}=\left(\frac{b-a}{4}\right)^{2},
$$

and this is our proposition. 
To illustrate the result (and to justify the title of the present note) we consider two examples of self-similar distributions $\mu$ with $S(\mu)=[0,1]$.

5.1 Riesz-Nagy distributions. Another example for singular measures investigated in [4] are the Riesz-Nagy singular distributions (more precisely, a special case of them). We need more information about them than given in the cited paper [4], so we recall the construction here.

Suppose $\left(\tau_{n}\right)_{n \geq 1}$ is a sequence of real numbers, $0<\tau_{n}<1$. Then we define inductively a sequence of continuous functions $F_{n}$ on $[0,1]$ as follows. Let $F_{0}(x)=\dot{x}$. For $n \geq 0$ define $F_{n+1}$ by

$$
\begin{array}{cl}
F_{n+1}\left(\frac{k}{2^{n}}\right)=F_{n}\left(\frac{k}{2^{n}}\right) & \left(k=0(1) 2^{n}\right) \\
F_{n+1}\left(\frac{2 k+1}{2^{n+1}}\right)=\frac{1-\tau_{n+1}}{2} F_{n}\left(\frac{k}{2^{n}}\right)+\frac{1+\tau_{n+1}}{2} F_{n}\left(\frac{k+1}{2^{n}}\right) & \left(k=0(1) 2^{n}-1\right) \\
F_{n+1}(x) \text { linear in the intervals }\left[\frac{k}{2^{n+1}}, \frac{k+1}{2^{n+1}}\right] & \left(k=0(1) 2^{n+1}-1\right) .
\end{array}
$$

Finally, we define the function $F$ by $F(x)=\lim _{n \rightarrow \infty} F_{n}(x)$. This limit function is continuous and strictly increasing, so there is a unique measure $\mu$ with distribution function $\mu((-\infty, x))=F(x)$. These measures are called Riesz-Nagy distributions.. In order to apply our results, we need the following

Lemma 8. If $\tau_{n} \leq 1-\delta<1$ for $n \geq 1$, then $S(\mu)=[0,1]$ and $\mu$ is regular.

Proof. Let

$$
I_{n k}=\left[\frac{k}{2^{n}}, \frac{k+1}{2^{n}}\right] . \quad\left(k=0,1, \ldots, 2^{n}-1\right)
$$

for $n \geq 0$. We show inductively $\mu\left(I_{n k}\right) \geq(\delta / 2)^{n}$ for all $0 \leq k \leq 2^{n}-1$. For $n=0$ this is trivial. Now we observe that the definition of $F_{n+1}$ implies

$$
F_{m}\left(\frac{k}{2^{n}}\right)=F_{n}\left(\frac{k}{2^{n}}\right) \quad\left(k=0,1, \ldots, 2^{n}\right)
$$

for all $m \geq n+1$ and thus $F\left(\frac{k}{2^{n}}\right)=F_{n}\left(\frac{k}{2^{n}}\right)$. Consequently, we have

$$
\mu\left(I_{n k}\right)=F_{n}\left(\frac{k+1}{2^{n}}\right)-F_{n}\left(\frac{k}{2^{n}}\right) .
$$

The definition of the measure $\mu$ now implies

and

$$
\mu\left(I_{n+12 k}\right)=\frac{1+\tau_{n+1}}{2} \mu\left(I_{n k}\right) \geq \frac{1-\tau_{n+1}}{2} \mu\left(I_{n k}\right) \geq \frac{\delta}{2} \mu\left(I_{n k}\right)
$$

and we are done.

$$
\mu\left(I_{n+12 k+1}\right)=\frac{1-\tau_{n+1}}{2} \mu\left(I_{n k}\right) \geq \frac{\delta}{2} \mu\left(I_{n k}\right)
$$

If $x \in[0,1]$ and $2^{-n} \leq r \leq 2^{-n+1}$, then the initerval $\Delta_{r}(x)$ contains at least one of the intervals $I_{n k}$, and it must be

$$
\mu\left(\Delta_{r}(x)\right) \geq\left(\frac{\delta}{2}\right)^{n} \geq\left(\frac{r}{2}\right)^{\alpha} \quad \text { where } \alpha=\frac{\log \frac{2}{\delta}}{\log 2} .
$$

This proves both the inclusion $x \in S(\mu)$ and the regularity of $\mu$. 
Application of our Theorem 2 gives the following have

Corollary 2. For the Riesz-Nagy distributions with $\tau_{n} \leq 1-\delta<1$ for $n \geq 1$ we

$$
\lambda_{n} \stackrel{d}{\longrightarrow} \frac{1}{16} \quad \text { and } \quad c_{n} \stackrel{d}{\longrightarrow} \frac{1}{2}
$$

This result is stronger than convergence of arithmetical means and it recovers the special case $\tau_{n} \equiv \dot{\tau} \dot{\epsilon}(0,1)$ considered in [4]. But nevertheless we feel that it is not strong enough - here we should have convergence in the usual sense.

5.2 An atomic measure. Our last example shows that the recurrence coefficients may behave quiet regularly even in the case of an atomic (or discrete) measure. We define

$$
\mu\left(\left\{\frac{m}{2^{n}}\right\}\right)=\frac{1}{3^{n}}
$$

for $n \geq 1, m$ odd and $0<m<2^{n}$, and $\mu(B)=0$ for any Borel set $B$ not containing a dyadic rational. The dyadic rationals are dense in $[0 ; 1]$; so we have $S(\mu)=[0,1]$. Any interval of length $2^{-n}$ contains a number of the form $m 2^{-n}$, and we easily prove $\mu\left(\Delta_{r}(x)\right) \geq(r / 2)^{\alpha}$ with $\alpha=\log 3 / \log 2$, i.e. we have the following

Lemma 9. The atomic measure $\mu$ is regular.

By Theorem 2 we obtain the following corollary (note that we have $c_{n} \equiv \frac{1}{2}$ by symmetry).

Corollary 3. For the measure defined above the coefficients $\lambda_{n}$ satisfy the convergence relation

$$
\lambda_{n} \stackrel{d}{\longrightarrow} \frac{1}{16}
$$

Again we feel that in fact the coefficients simply converge to $\frac{1}{16}$. This is suggested by numerical experiments: The measure has a simple self-similar structure, and one easily shows that

$$
\int f(x) d \mu(x)=\frac{1}{3}\left(f\left(\frac{1}{2}\right)+\int f\left(\frac{x}{2}\right) d \mu(x)+\int f\left(\frac{x+1}{2}\right) \cdot d \mu(x)\right)
$$

for any bounded function $f$. From this equation we can derive simple recurrences not only for ordinary moments $\int x^{n} d \mu(x)$, but for modified moments $\int T_{n}^{*}(x) d \mu(x)$ too, where $T_{n}^{*}(x)=T_{n}(2 x-1)$ are the shifted Chebyshev polynomials of first kind. With a variant of the modified Chebyshev algorithm (see [3]) we can calculate numerically some $\lambda_{n}$ beyond the $n=18$ in [4] (actually up to $n=5000$ ), and the algorithm seems to be stable. The values

$$
\varepsilon_{n}=\max _{2^{n-1}<k \leq 2^{n}}\left|\lambda_{k}-\frac{1}{16}\right|
$$


should give some feeling for the long-range behaviour of the coefficients $\lambda_{n}$ :

\begin{tabular}{r|c}
$n$ & $\varepsilon_{n}$ \\
\hline 4 & $3.05432773230336 \mathrm{E}-0002$ \\
5 & $2.71889842287010 \mathrm{E}-0002$ \\
6 & $2.04401383587083 \mathrm{E}-0002$ \\
7 & $1.79923599931158 \mathrm{E}-0002$ \\
8 & $1.46869431344498 \mathrm{E}-0002$ \\
9 & $1.14468494701327 \mathrm{E}-0002$ \\
10 & $9.76190450455761 \mathrm{E}-0003$ \\
11 & $7.55169329744376 \mathrm{E}-0003$ \\
12 & $6.23379099249632 \mathrm{E}-0003$
\end{tabular}

Indeed, this figures (and rather similar results for the Riesz-Nagy distribution with $\tau_{n} \equiv \frac{1}{2}$ ) suggest convergence. Unfortunately, the author has no rigorous proof of their reliability.

\section{References}

[1] Bessis, D.: Orthogonal polynomials, Padé approximations and Julia sets. In: Orthogonal Polynomials, Theory and Practice. NATO ASI Series C: Vol. 294 (ed.: P. Nevai). Dortrecht etc.: Kluwer Acad. Publ. 1990, pp. 55 - 97.

[2] Chihara, T. S.: An Introduction to Orthogonal Polynomials. New York: Gordon \& Breach 1978.

[3] Gautschi, W.: Computational aspects of orthogonal polynomials. In: Orthogonal Polynomials, Theory and Practice. NATO ASI Series C: Vol. 294 (ed.: P. Nevai). Dortrecht etc.: Kluwer Acad. Publ. 1990, pp. 181 - 216.

[4] Goh, W. and J. Wimp: Asymptotics for the moments of singular distributions. J. Appr. Theory 74 (1993), $301-334$.

[5] Mercer, A. McD.: A note on the zero distribution of orthogonal polynomials. J. Appr. Theory $64(1991), 238-242$.

[6] Nevai, P. G.: Orthogonal Polynomials. Mem. Amer. Math. Soc. 18 (1979), 1 - 185.

[7] Saff, E. B.: Orthogonal polynomials from a complex perspective. In: Orthogonal Polynomials, Theory and Practice. NATO ASI Series C: Vol. 294 (ed.: P. Nevai). Dortrecht etc.: Kluwer Acad. Publ. 1990, pp. 363 - 393.

[8] Stahl, H. and V. Totik: N th root asymptotic behavior of orthonormal polynomials. In: Orthogonal Polynomials, Theory and Practice. NATO ASI Series C: Vol. 294 (ed.: P. Nevai). Dortrecht etc.: Kluwer Acad. Publ. 1990, pp. $395-417$.

[9] Ullman, J. L.: On the regular behaviour of orthogonal polynomials. Proc. London Math. Soc. 24 (1972), $119-148$. 